\title{
Movimiento de las interfases durante la solidificación horizontal de aleaciones estaño-zinc
}

\author{
Movement of the interphases during the \\ horizontal solidification of tin-zinc alloys
}

Alex Iván Kociubzyk ${ }^{1,2}$, Roberto Samuel Rozicki ${ }^{2}$, Alicia Esther Ares ${ }^{1,2}$

\footnotetext{
${ }^{1}$ Instituto de Materiales de Misiones - IMAM - CONICET/UNaM - CP: 3300, Posadas, Misiones, Argentina.

${ }^{2}$ Facultad de Ciencias Exactas, Químicas y Naturales - UNaM - CP: 3300, Posadas, Misiones, Argentina. e-mail: akociubczyk@gmail.com; roberto.rozicki@gmail.com; aares@fceqyn.unam.edu.ar
}

\section{RESUMEN}

El procesamiento por solidificación es una de las rutas más importantes para producir materiales, especialmente metales y aleaciones. Los parámetros para la transformación de líquido a sólido, tales como, el gradiente de temperatura, la velocidad de crecimiento, la velocidad de enfriamiento, la velocidad de la interfase, varían de un proceso a otro y en un solo proceso también varían como una función del tiempo y de la posición. Estas variaciones junto con las diferentes composiciones de las aleaciones, conducen a una multitud de microestructuras y, por lo tanto, afectan al comportamiento del material.

En el presente trabajo se estudia la evolución de las interfases en las aleaciones Sn-Zn (Sn-6\%Zn (hipoeutéctica) y Sn-15\%Zn (hipereutéctica)) cuando son solidificadas en forma unidireccional horizontal mediante la extracción calórica en dos sentidos opuestos. Se obtuvieron probetas de aleaciones Sn-Zn con forma de segmentos cilíndricos, con una longitud promedio de entre $13 \mathrm{~cm}-17 \mathrm{~cm}$, un ancho promedio de entre 3 $\mathrm{cm}-3,2 \mathrm{~cm}$ y un espesor promedio de entre $0,8 \mathrm{~cm}-1,2 \mathrm{~cm}$, dependiendo de la experiencia.

Se determinaron los parámetros térmicos (velocidades de enfriamiento, gradientes de temperatura, posiciones y velocidades de las interfases presentes) y se relacionaron con las estructuras finales producto de la solidificación. Se encontró que el valor de la velocidad de las interfases es máximo cuando chocan los frentes opuestos de solidificación, avanzando en direcciones opuestas en las probetas. Los valores de las velocidades de las interfases fueron superiores a $2 \mathrm{~mm} / \mathrm{s}$ para ambas aleaciones $\mathrm{Sn}-\mathrm{Zn}$ analizadas.

Palabras clave: aleaciones estaño-zinc, solidificación direccional, parámetros térmicos.

\section{ABSTRACT}

Solidification processing is one of the important routes to produce materials, especially metals and alloys. The parameters for the transformation from liquid to solid, such as the temperature gradient, the growth rate, the cooling rate, the interphase velocity, vary from process to process and in one process also as a function of time and space. These variations together with the different alloy compositions, lead to a multitude of microstructures and therefore, affect the behavior of the material.

In this work the evolution of the interphases is studied in Sn-Zn (Sn-6wt.\% Zn (hypoeutectic) and Sn$15 \mathrm{wt} . \% \mathrm{Zn}$ (hypereutectic)) when the specimens were unidirectionally horizontally solidified through heat extraction in two opposite directions. Sn-Zn specimens shaped in cylindrical segments were obtained, with an average length between $13 \mathrm{~cm}-17 \mathrm{~cm}$, an average width between $3 \mathrm{~cm}-3.2 \mathrm{~cm}$ and an average thickness of $0.8 \mathrm{~cm}-1.2 \mathrm{~cm}$, depending on the experience.

The thermal parameters (cooling rates, temperature gradients, positions and velocities of the present interfaces) were determined and related to the final structures resulting from the solidification. It was found that the value of the velocity of the interphase is maximum when opposing fronts of solidification collide, advancing in opposite directions in the specimens. The values were superior to $2 \mathrm{~mm} / \mathrm{s}$ for both analyzed SnZn alloys. 
Keywords: tin-zinc alloys, directional solidification, thermal parameters.

\section{INTRODUCCIÓN}

El estaño se encuentra en muchas aleaciones y se usa para recubrir otros metales protegiéndolos de la corrosión. Las aleaciones base estaño son ampliamente utilizadas en la fundición, en aleaciones base bronce, en aleaciones con plomo, antimonio, cobre, zinc, bismuto, fósforo, en aleaciones Babbitt, en revestimientos, en soldaduras blandas. Hasta la actualidad el estaño es aleado con el zinc y con el plomo para elaborar metales de soldadura blanda, que son metales con un punto de fusión relativamente bajo, y que se utilizan para unir componentes eléctricos, tuberías, y otros ítems metálicos [1,2].

En el área de solidificación resulta de suma importancia la determinación de los parámetros macroestructurales, de los parámetros que afectan al proceso de solidificación y el acoplamiento de ambas determinaciones, junto con el hecho de que sólo mediante la validación experimental, los modelos de solidificación se pueden aplicar con la suficiente confianza [3-12].

Durante la solidificación, el calor latente de transformación del líquido al sólido es liberado durante la nucleación y el crecimiento de los primeros núcleos de sólido. La cantidad de energía liberada depende del tipo y de la cantidad de cristales en formación en función del tiempo. La cantidad de energía emitida puede ser suficiente para detener el enfriamiento del metal, disminuyendo su velocidad de enfriamiento [3]. Estos eventos energéticos asociados a la solidificación pueden ser analizados mediante el análisis térmico a partir de las curvas de enfriamiento obtenidas experimentalmente en el interior del metal durante la solidificación. Se suele suponer que la transición de estructura columnar a equiaxial (TCE) se produce cuando el avance del frente columnar es bloqueado por los granos equiaxiales que crecen en el líquido constitucionalmente subenfriado, delante de las dendritas columnares [3, 7-12].

En investigaciones anteriores, con probetas sometidas a solidificación direccional horizontal, se determinó que la extracción de calor resultó en dos direcciones opuestas, dando lugar a seis interfases de solidificación [11-16]. Como fue descripto por GUEIJMAN et al. en [11], en los trabajos se realiza el análisis de las interfases (no interfaces).

El objetivo del presente trabajo fue el de solidificar direccionalmente las aleaciones Sn-Zn ( $\mathrm{Sn}-6 \% \mathrm{Zn}$ (hipoeutéctica) y Sn-15\%Zn (hipereutéctica)) mediante un proceso de solidificación direccional horizontal, con dos sentidos opuestos de extracción de calor. Asimismo, determinar los parámetros térmicos involucrados en el proceso y correlacionarlos con las macroestructuras obtenidas. Asimismo, analizar las posiciones y velocidades de las interfases presentes durante la solidificación y su vinculación con la posición de la contracción final que se produce en las probetas.

\section{MATERIALES Y MÉTODOS}

La solidificación direccional de las aleaciones se realizó en un horno de solidificación direccional horizontal que consta de una unidad de calentamiento y de sistemas de control y de adquisición de temperaturas, además de un sistema de extracción calórica direccional. Un esquema básico del dispositivo experimental se puede observar en la Figura 1 (a) y una foto de probeta obtenida se presenta en la Figura 1 (b). Se obtuvieron probetas de aleaciones Sn-Zn con forma de segmentos cilíndricos, con una longitud promedio de entre $13 \mathrm{~cm}-17$ $\mathrm{cm}$, un ancho promedio de entre $3 \mathrm{~cm}-3,2 \mathrm{~cm}$ y un espesor promedio de entre $0,8 \mathrm{~cm}-1,2 \mathrm{~cm}$, dependiendo de la experiencia.

Se solidificaron por triplicado el $\mathrm{Sn}$ y el $\mathrm{Zn}$ de pureza comercial y las aleaciones Sn-Zn de cada composición (Zn-6\%Zn y Sn-15\%Zn, porcentajes en peso) por solidificación direccional horizontal, con dos sentidos de extracción calórica. Para la preparación de las aleaciones, los elementos puros (Zn y Sn) se fusionaron en moldes de grafito en un horno de mufla, y luego se colocaron en el molde de arcilla, dentro del horno horizontal, para la solidificación direccional con extracción de calor en dos direcciones opuestas (ver la Figura 1 (a)). A continuación, las muestras se desbastaron con papel abrasivo de $\mathrm{SiC}$ de diferentes tamaños de grano, desde \# 60 hasta \# 1500. Para observar la macroestructura, las probetas fueron sometidas a un ataque químico que consistía en exponerlas a una solución de $\mathrm{HCl}$ al 36,5\%, a temperatura ambiente (entre 5 y 30 segundos). Esto permitió definir zonas de diferentes estructuras a lo largo de la muestra: una zona columnar de granos grandes y alargados creciendo desde los extremos hacia el centro y la zona de granos equiaxiales en el centro de las probetas (Figura 1 (c)). 


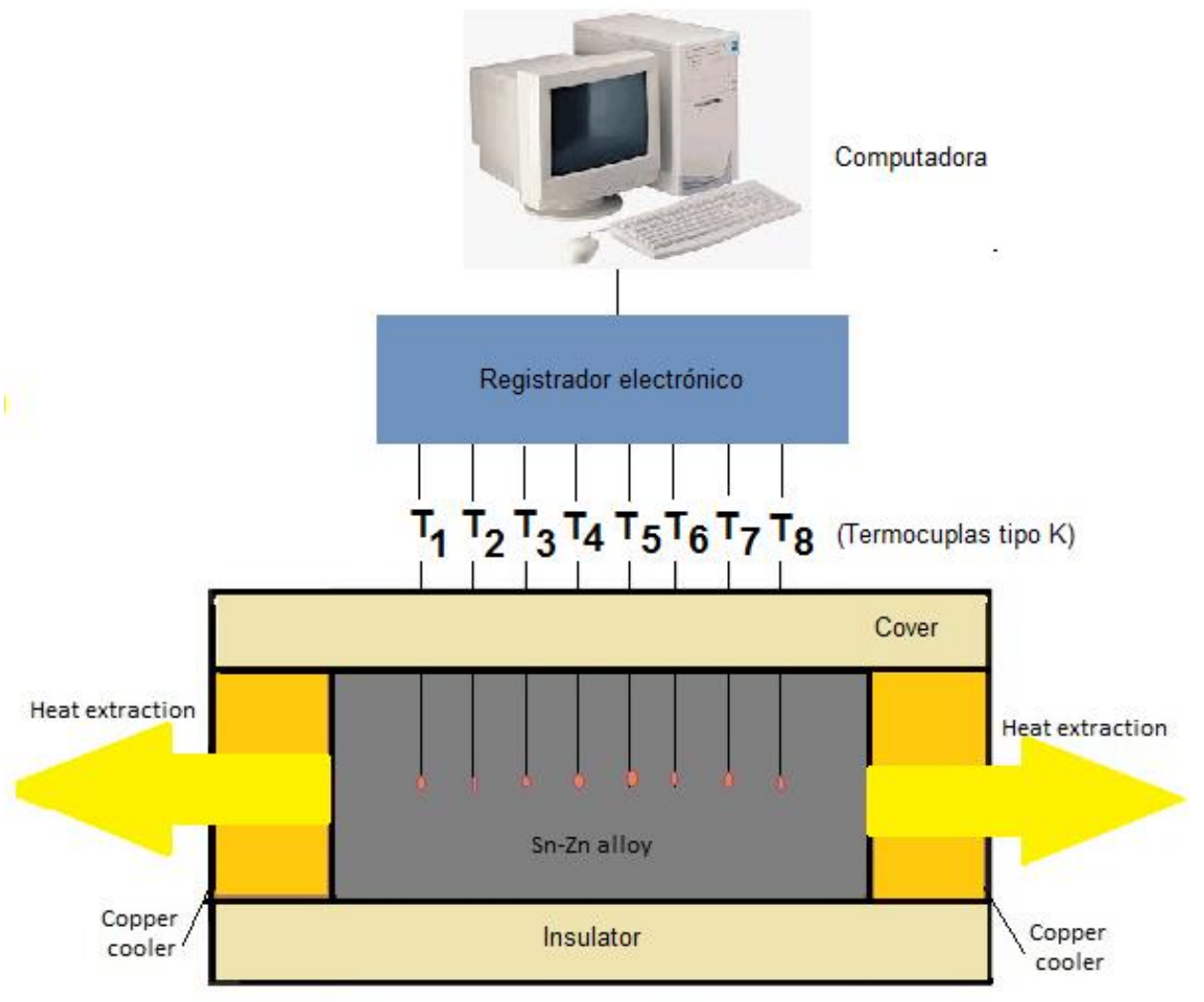

(a)

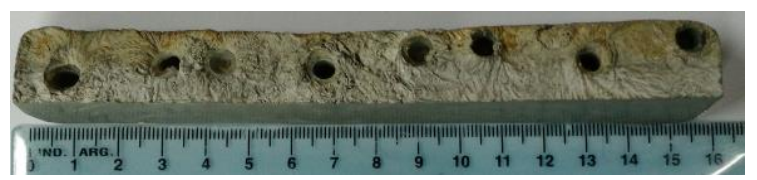

(b)

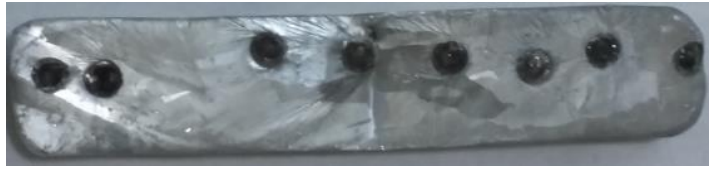

(c)

Figura 1: (a) Esquema del dispositivo experimental de solidificación unidireccional horizontal empleado. (b) Probeta de Sn-6\% Zn obtenida (los agujeros redondos indican las posiciones en las que se insertaron las termocuplas). (c) Macroestructura típica mostrando em el centro la colisión de ambos frentes.

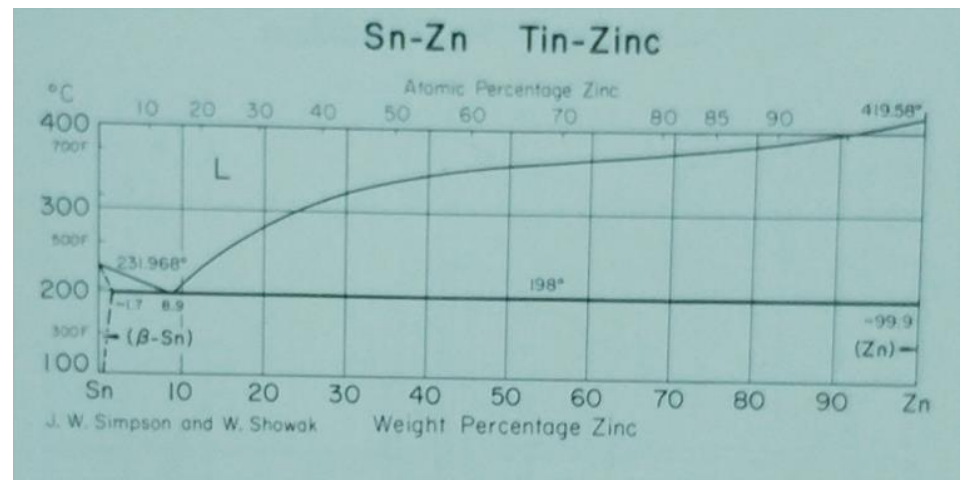

Figura 2: Diagrama de fases Sn-Zn [2]. 


\section{RESULTADOS Y DISCUSIÓN}

Graficando los datos de la temperatura en función del tiempo (Figura 3), se observó la presencia de tres zonas en el gráfico: primero - un período de enfriamiento de la masa fundida (temperatura de sobrecalentamiento y la temperatura de liquidus); Segundo - un periodo de solidificación (entre la temperatura de liquidus y la temperatura eutéctica); Tercero - un período de enfriamiento del sólido (por debajo de la temperatura eutéctica) $[12-14]$.

El valor medio de la temperatura eutéctica registrada fue de $198,5^{\circ} \mathrm{C}$ (en el diagrama de equilibrio de la Figura 2 se puede observar que el valor teórico es de $198^{\circ} \mathrm{C}$ ).

A partir de los datos de temperatura registrados y procesados, se obtuvieron los parámetros térmicos tales como, el tiempo de solidificación local en la posición de cada termocupla, los gradientes de temperatura (ver Figura 4) y las velocidades de enfriamiento (ver Figura 5).

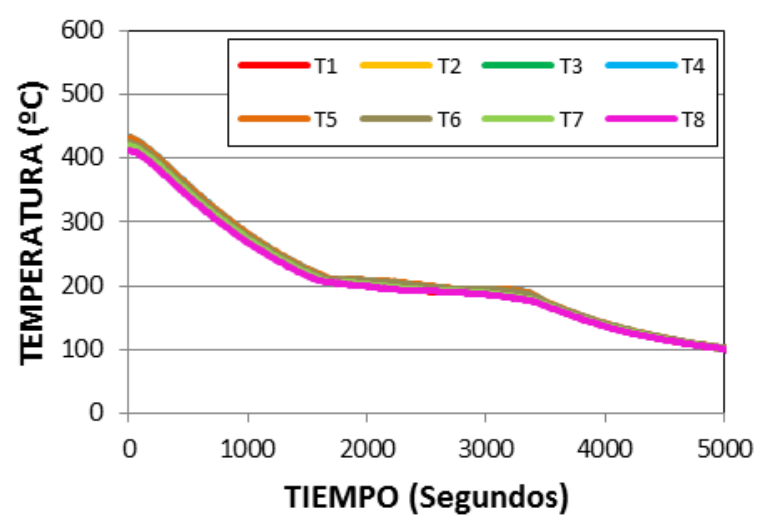

(a)

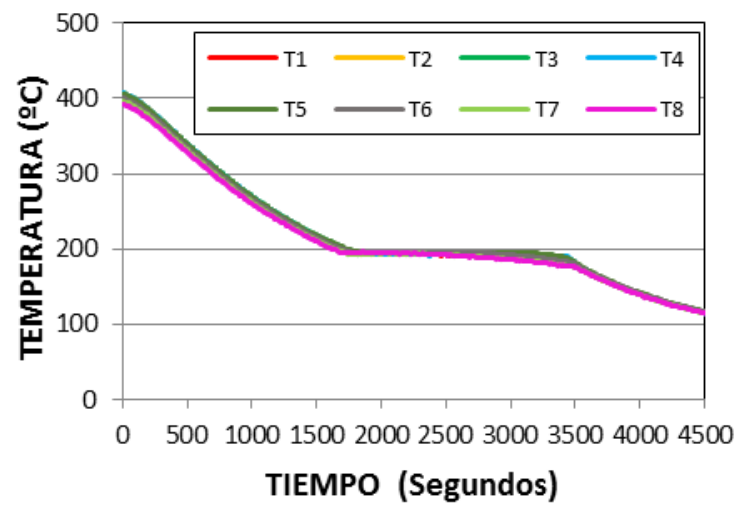

(b)

Figura 3: Temperatura versus tiempo (T1 y T8 son las termocuplas posicionadas em ambos extremos de las probetas, ver el esquema de la Figura 1). (a) Sn-6\%Zn. (b) Sn-15\%Zn.

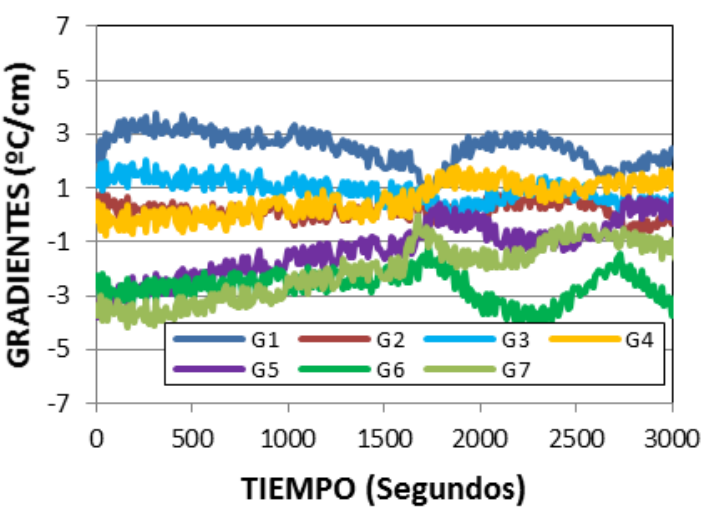

(a)

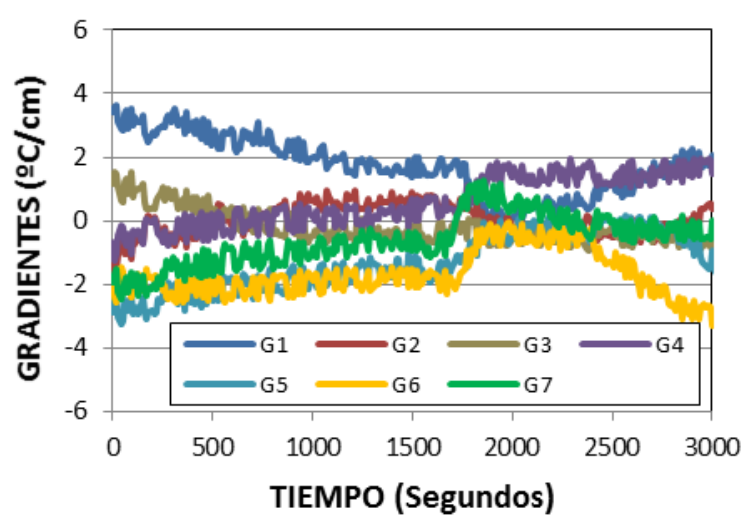

(b)

Figura 4: Gradientes de temperatura versus tiempo (en la posición de cada termocupla). (a) Sn-6\%Zn. (b) Sn-15\% Zn (los valores de gradientes negativos corresponden a los calculados a partir del extremo izquierdo de las probetas).

A partir del análisis de las gráficas, se observa que en el caso de las probetas con estructuras equiaxiales, los gradientes de temperatura desde el principio tienen valores bajos $\left(<5{ }^{\circ} \mathrm{C} / \mathrm{cm}\right)$ y no hay gradientes mínimos y críticos de modo que se produzca una transición en la estructura de los granos. Se obtuvo un perfil de comportamiento similar de los gradientes de temperatura en el caso de las probetas con estructura completamente columnar 


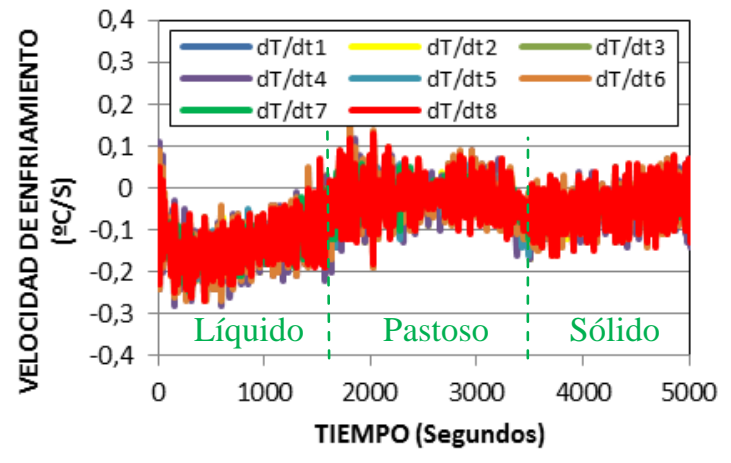

(a)

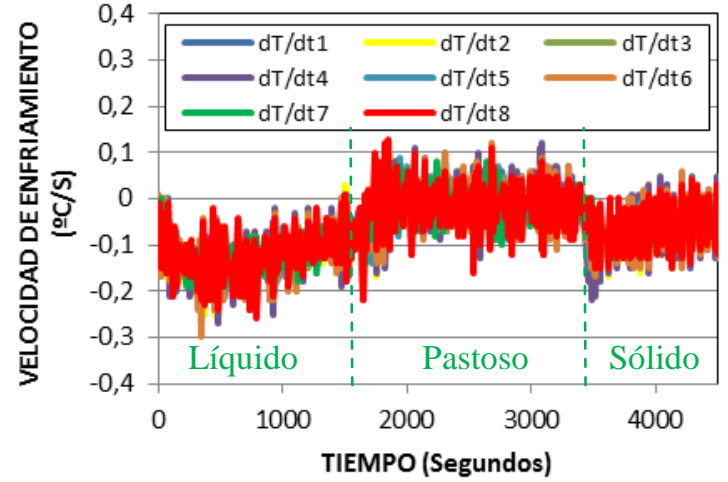

(b)

Figura 5: Variación de la velocidad de enfriamiento versus tiempo (em la posición de cada termocupla). (a) Sn-6\%Zn. (b) Sn-15\% Zn.

Después de obtener los datos de temperaturas en función del tiempo para la aleación en cuestión, como se indica en las Figuras 3, se determinaron las velocidades de enfriamiento en las aleaciones líquida, pastosa y sólida tomando los valores de la derivada de temperatura con respecto al tiempo. Para cada posición de termocupla considerada como cocientes de diferencias centradas, como se muestra en las Figuras 5. A partir de los cambios en las derivadas de las velocidades de enfriamiento, los momentos aproximados del inicio y fin de la solidificación en el volumen de detección en que cada termocupla (instante de la interfase líquida $[\mathrm{L} /(\mathrm{L}+\mathrm{S})]$ o instante de la interfase sólida, $[(\mathrm{S}+\mathrm{L}) / \mathrm{S}])$, pasa a través de la posición donde se encuentra la termocupla]). A medida que cada probeta se enfría predominantemente desde ambos extremos, es posible determinar un par de interfases que avanzan para cada tipo de interfase ([L / $(\mathrm{L}+\mathrm{S})]$ o $[(\mathrm{S}+\mathrm{L}) / \mathrm{S}]$, es decir, dos interfases líquidas y dos interfases sólidas. Cada par de interfases del mismo tipo se mueven en la dirección longitudinal pero com direcciones opuestas de movimiento.

En la Figura 6 se muestran las probetas y macroestructuras obtenidas para ambas aleaciones. En las fotos se pueden apreciar los agujeros producto del posicionamiento de las termocuplas durante la solidificación, además de las zonas en las que se produjo el choque de los frentes de solidificación avanzando en direcciones opuestas, lo cual coincide com las zonas em donde se observan las contracciones finales por la solidificación.

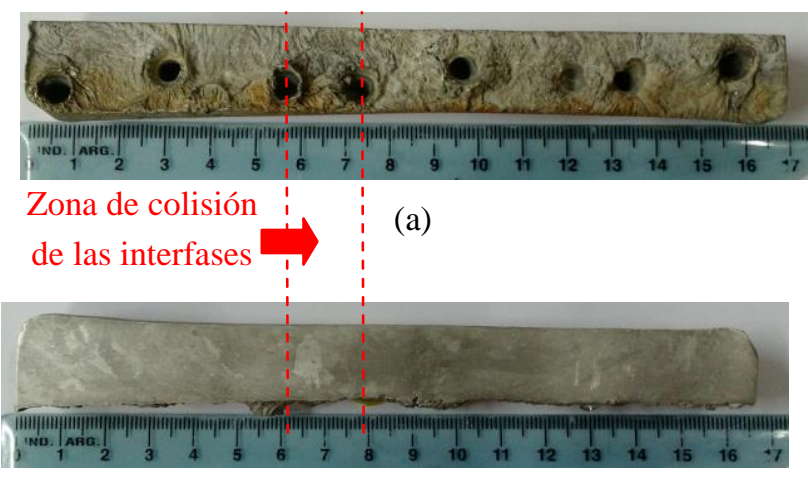

(b)

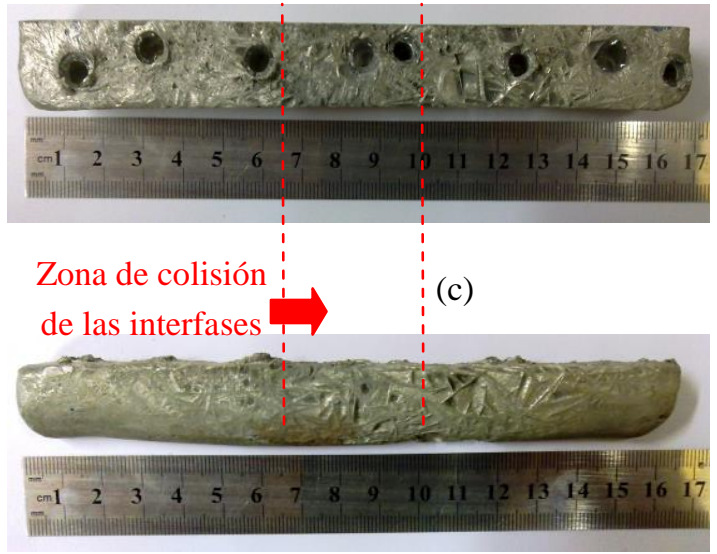

(d)

Figura 6: (a) y (c) Probetas de Sn-6\%Zn y Sn-15\%Zn. (b) y (d) Macrografìas de Sn-6\%Zn y Sn-15\%Zn.

La Figura 7 muestra la posición de las interfases líquidas ó $[\mathrm{L} /(\mathrm{L}+\mathrm{S})]$ y sólidas ó $[(\mathrm{S}+\mathrm{L}) / \mathrm{S}]$ durante la solidificación de la probeta em función del tiempo. Se puede ver que, aunque cada par de interfases del mismo tipo está representado por una sola línea, cada línea corresponde a dos interfases del mismo tipo, debido a que cada interfase no puede estar en dos puntos en el espacio en el mismo instante. 


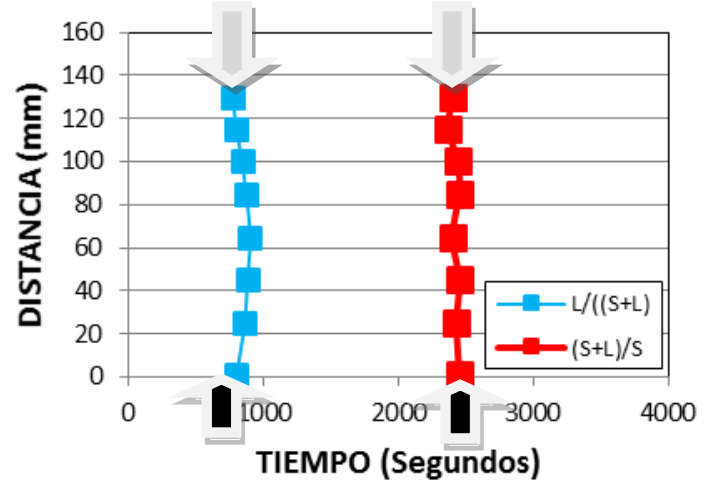

(a)

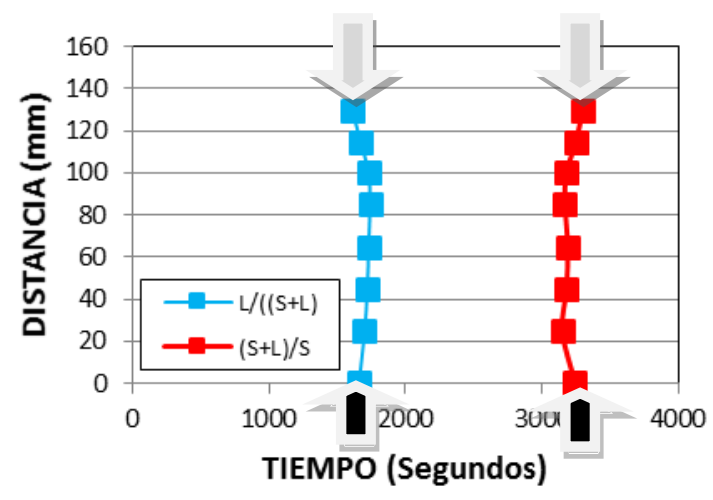

(b)

Figura 7: Posición de las interfases em función del tiempo. (a) Sn-6\%Zn. (b) Sn-15\%Zn.

El instante de colisión de dos interfases del mismo tipo se caracteriza por el máximo momento en la posición considerada de las curvas como punto "C" [11]. Así, en las curvas de la Figura 7 se representan cuatro interfases, pero sólo se muestran dos curvas. Cada curva corresponde a un par de interfases que avanzan en direcciones opuestas y que chocan en algún punto dentro de la probeta (flechas grises y negras). Obsérvese que los puntos de colisión de las interfases o puntos de las diferentes curvas "C" no coinciden en la misma posición de la probeta, que es el máximo de la curva tiempo vs. posición (t vs. x). La gráfica de la velocidad de la interfase liquidus, $\mathrm{V}_{\mathrm{L}}$, versus la posición media en la probeta se muestra en la Figura 8 . El valor máximo en este gráfico corresponde a la posición de colisión de ambas interfases en la probeta. A partir de ciertas funciones $\mathrm{x}$ vs $\mathrm{t}$, los puntos de datos de cada una de las interfases se pueden identificar como pertenecientes a la interfase dada (derecha o izquierda) que se mueven en cada una de las direcciones. Los puntos extremos no se consideraron en los ajustes, porque llas termocuplas más extremas $\mathrm{T}_{1}$ y $\mathrm{T}_{8}$ son sólo representativos de medio volúmenes en cuestión, pero sirven como puntos de comparación con extrapolaciones de los valores de función determinados. La derivada de estas funciones corresponde a la inversa de la velocidad de las interfases, tratada como una curva única. En los gráficos de las Figuras 9 se muestran los valores calculados de $\mathrm{V}^{-1}$ a partir de polinomios construidos de acuerdo con la posición (expresada en función del tiempo de solidificación local). Los valores positivos de $\mathrm{V}^{-1}$ representan interfases que se mueven de izquierda a derecha, y los valores negativos de $\mathrm{V}^{-1}$ son interfases que se mueven de derecha a izquierda. El tiempo máximo en cada gráfica corresponde al instante de colisión de dos interfases de avance opuesto. Esta colisión de interfases deja a la formación de vacíos, poros y defectos internos en las probetas (zonas marcadas entre las líneas de trazos rojas las Figuras 6).

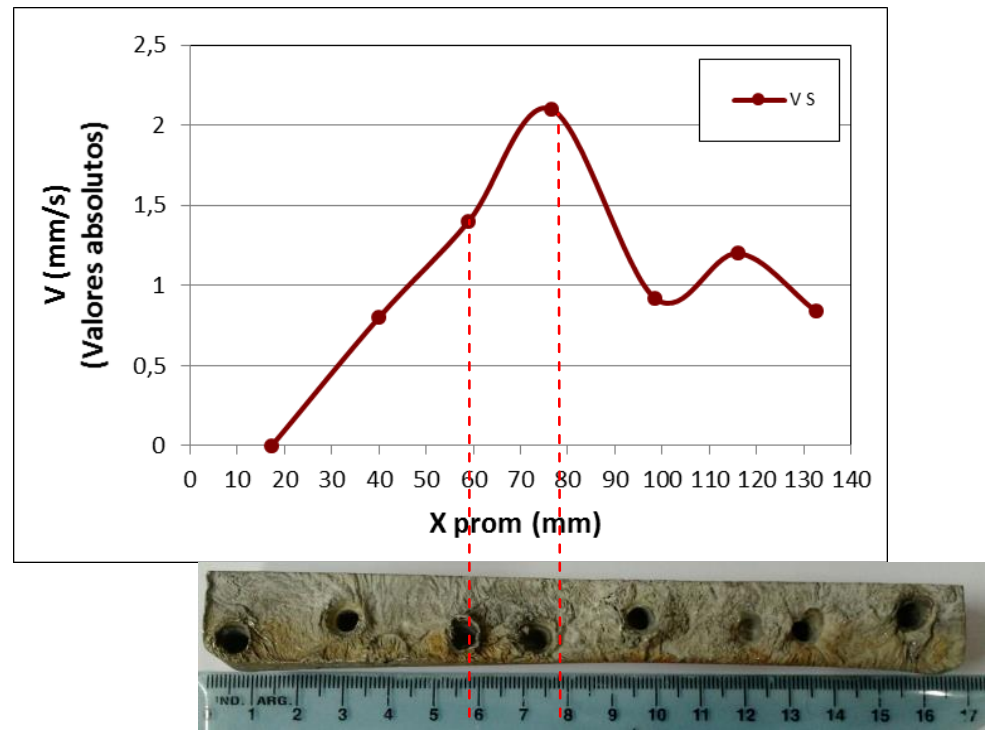




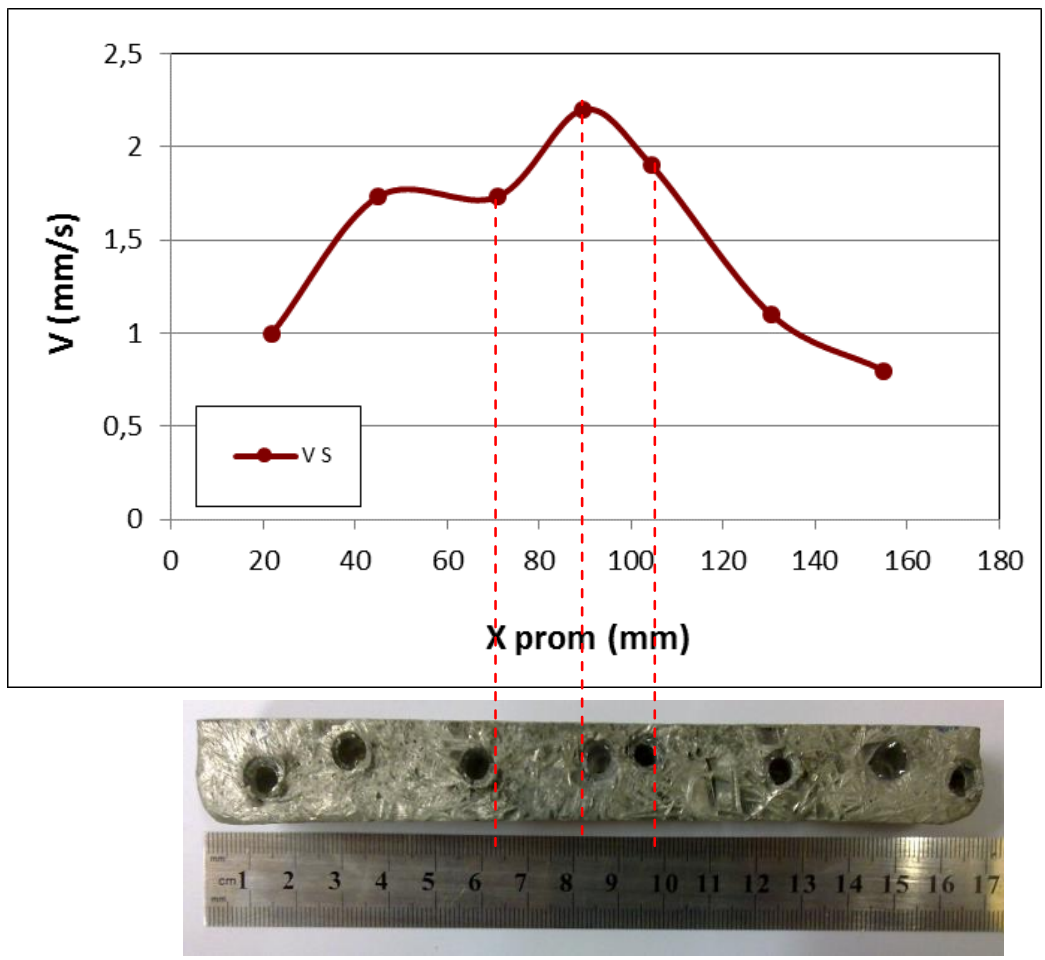

(b)

Figura 8: Velocidad absoluta de la interfase sólida versus posición. (a) Sn-6\%Zn. (b) Sn-15\%Zn.

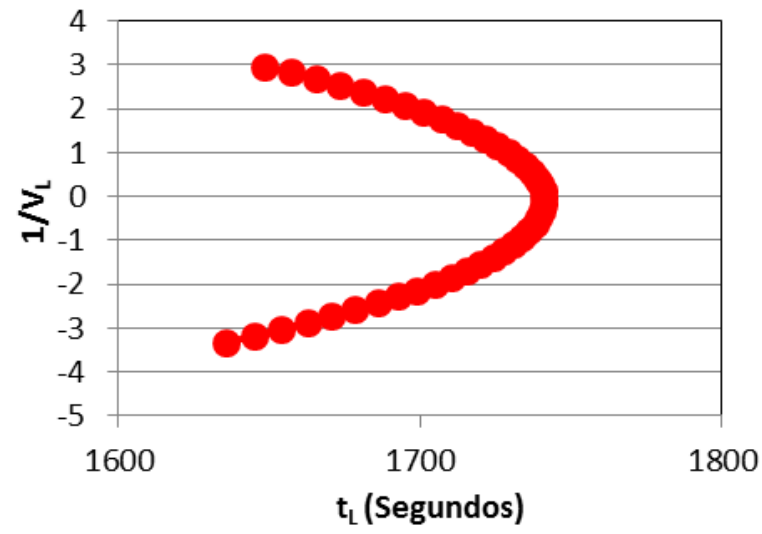

(a)

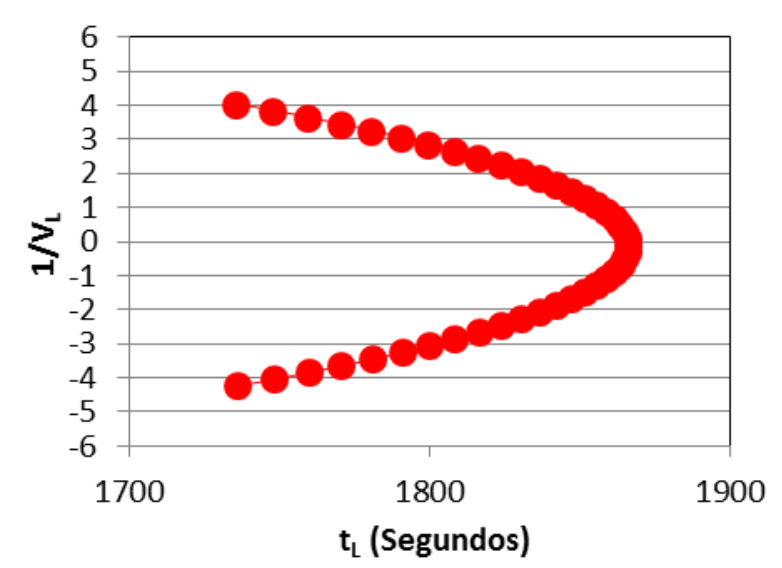

(b)

Figura 9: $\mathrm{V}^{-1}$ versus tiempo de solidificación local, $\mathrm{t}_{\mathrm{L}}$. (a) $\mathrm{Sn}-6 \% \mathrm{Zn}$. (b) $\mathrm{Sn}-15 \% \mathrm{Zn}$.

En la Figura 8 se observa que los valores de las velocidades de las interfases fueron superiores a 2 $\mathrm{mm} / \mathrm{s}$ para ambas aleaciones $\mathrm{Sn}-\mathrm{Zn}$ analizadas.

Los valores de velocidad de las interfases, $\mathrm{V}^{-1}$, en función del tiempo de solidificación local, $\mathrm{t}_{\mathrm{L}}$, se grafican en la Figura 9. Como se mencionó previamente para otras aleaciones [11-16], el tiempo máximo en el gráfico corresponde al tiempo de la colisión de dos frentes opuestos. El valor máximo de las velocidades de las interfases, que avanzan en sentidos opuestos, coinciden con la zona de colisión de ambos frentes en solidificación en las probetas, y además, con la zona de contracción final de las mismas, como se observa en las macrografías de la Figura 8. 


\section{CONCLUSIONES}

Los experimentos de solidificación direccional horizontal se realizaron con dos direcciones de extracción de calor, en las aleaciones $\mathrm{Sn}-\mathrm{Zn}$ hipoeutécticas (Sn-6\%Zn) y hipereutécticas ( $\mathrm{Sn}-15 \% \mathrm{Zn})$.

Se determinaron los principales parámetros, a saber: a) el inicio y el final de la solidificación en cada posición considerada en las probetas, b) el tiempo de solidificación local (determinado por los cambios de pendientes en las curvas de temperatura versus tiempo), c) las velocidades de enfriamiento (calculadas como la derivada de las curvas $\mathrm{T}$ versus $\mathrm{t}$ ), d) las velocidades de las interfases, que avanzan en direcciones opuestas e) el valor de la velocidad de las interfases cuando chocan los frentes opuestos de solidificación, avanzando en direcciones opuestas en las probetas (los valores de las velocidades de las interfases fueron superiores a 2 $\mathrm{mm} / \mathrm{s}$ para ambas aleaciones $\mathrm{Sn}-\mathrm{Zn}$ analizadas).

El valor máximo de las velocidades de las interfases, que avanzan en sentidos opuestos, coinciden con la zona de colisión de ambos frentes en solidificación en las probetas, y además, con la zona de contracción final de las mismas.

\section{AGRADECIMIENTOS}

Los autores agradecen al Consejo Nacional Investigaciones Científicas y Técnicas (CONICET) y a la Agencia Nacional de Promoción Científica y Tecnológica (ANPCyT), ambos de Argentina, por el financiamiento otorgado para realizar la presente investigación.

\section{BIBLIOGRAFÍA}

[1] KALPAKJIAN, S., SCHMIDT, S.R., Manufactura, ingeniería y tecnología, Pearson Educación, México, 2008.

[2] ASM HANDBOOK COMMITTEE, Metals Handbook, American Society for Metals, Metals Park Ohio, United States, 1984.

[3] SPITTLE, J. A., Columnar to equiaxed grain transition in as solidified alloys", International Materials Reviews, v. 51, pp. 247-269, Agosto 2006.

[4] BADILLO, A., BECKERMANN, C., "Phase-field simulation of the columnar-to-equiaxed transition in alloy solidification", Acta Materialia, v. 54, pp. 2015-2026, 2006.

[5] McFAdDEN, S., BROWNE, V, GANDIN, C. A., "A Comparison of columnar-to-equiaxed transition prediction methods using simulation of the growing columnar front", Metall. Mater. Trans. A, v. 40, pp. 662672, Enero 2009.

[6] REINHART, G., MANGELINCK-NOËL, N., NGUYEN-THI, H., et al., "Investigation of columnarequiaxed transition and equiaxed growth of aluminium based alloys by X-ray radiography”, Mater Sci Eng A, vol. 413-414, pp. 384-388, Diciembre 2005.

[7] GANDIN, C. A. "From constrained to unconstrained growth during directional solidification", Acta Mater, vol. 48, pp. 2483-2501, Marzo 2000.

[8] AGALIOTIS, E. M., SCHVEZOV, C.E., ROSENBERGER, M.R., et al., "A numerical model study of the effect of interface shape on particle pushing", Journal of Crystal Growth, v. 354, pp. 49-56, Septiembre 2012.

[9] ARES, A. E., SCHVEZOV, C.E., "Solidification parameters during the columnar-to-equiaxed transition in lead-tin alloys", Metall. Mater. Trans. A, v. 31, pp. 1611-1625, Junio 2000.

[10] ARES, A. E., SCHVEZOV, C.E., "Influence of solidification thermal parameters on the columnar-toequiaxed transition of aluminum-zinc and zinc-aluminum alloys", Metall. Mater. Trans. A, v. 38, pp. 14851499, Julio 2007.

[11] GUEIJMAN, S. F., SCHVEZOV, C. E., ARES, A. E. "Solidification and characterization of Zn-Al and Zn-Ag diluted alloys", Materials Transactions, v. 51, pp. 1851-1870, Agosto 2010.

[12] GUEIJMAN, S. F., SCHVEZOV, C. E., ARES, A. E., "Theory and experiments of interphase formation and structure in horizontal directionally solidified Zn-Al dendritic alloys", Crystal Growth: Theory, Mechanisms and Morphology, pp. 263-291, 2012.

[13] ZURKO, M., RODRIGUEZ, C. M., SCHVEZOV, C. E., MENDEZ, C. M., et al., "Horizontal directional solidification of Zn-Al alloys", TMS Annual Meeting, v.3, pp. 153-160, Febrero 2012. 
[14] DESROSIN, W., SCHVEZOV, C. E., ARES, A. E., "Thermal and metallographic parameters evolution during solidification of Zn-Sn alloys”, TMS Annual Meeting, pp. 501-508, Febrero 2014.

[15] KOCIUBCZYK, A.I., DESROSIN, W., BOYCHO, L.M., et al., "Interphase evolution and defect formation during horizontally directional solidification process of Sn-Zn alloys", TMS Annual Meeting, pp. 5764, Febrero 2015.

[16] KOCIUBCZYK, A.I., ROZICKI, R., SCHEIBER, V.L., et al., "Solidification and evaluation of thermal parameters of Sn-Zn eutectic alloys horizontally solidified”, TMS Annual Meeting, pp. 97-104, Febrero 2016. 